\title{
A Unified Specification Format for Interlevel Relations between Agent Models in Multiple Abstraction Dimensions ${ }^{1}$
}

\author{
Jan Treur \\ VU University Amsterdam, Department of Artificial Intelligence \\ De Boelelaan 1081, 1081 HV Amsterdam, The Netherlands \\ j.treur@vu.nl_http://www.few.vu.nl/ treur
}

\begin{abstract}
Multiagent systems for a certain application area can be modelled at multiple levels of abstraction. Interlevel relations are a means to relate models from different abstraction levels. Three dimensions of abstraction often occurring are the process abstraction, temporal abstraction, and agent cluster abstraction dimension. In this paper a unifying formalisation is presented that can be used as a framework to specify interlevel relations for any of such dimensions. The approach is illustrated by showing how a variety of different types of abstraction relations between multi-agent system models can be formally specified in a unified manner.
\end{abstract}

\section{Introduction}

Different models describing the same process in reality, usually are based on different conceptualisations of this process. As they are models of the same process in which different concepts used may refer to the same aspects or elements in reality, a natural question is how in general two of such descriptions can be related to each other. In the literature on reduction relations it is described, for example, how by an interlevel relation a functional or cognitive model (or theory) can be related to a biochemical or neurological model, (e.g., [5], [20]). In [29] this notion was further developed by formalising (context-dependent) interlevel relations between cognitive agent models and neurological agent models. More in general, abstraction levels between multiagent system models may concern different dimensions of abstraction. For example, process abstraction can be considered from neurological to cognitive models (as addressed by the literature on reduction relations), and from cognitive to behavioural models (as is done in information hiding or from a behaviourist perspective; e.g., [4]). As another example, in temporal abstraction the detailed steps of a process are left out of consideration and instead the patterns are considered emerging from such steps. Yet another example is agent cluster abstraction: abstracting from individual

\footnotetext{
${ }^{1}$ A preliminary, shorter version of this paper was presented at the $24^{\text {th }}$ International Conference on Industrial, Engineering and Other Applications of Applied Intelligent Systems (IEA/AIE 2011).
} 
agents to consider populations, groups or clusters of agents instead, as often happens in organisational modelling, ecological modelling, and system dynamics.

In [8] a three-dimensional abstraction framework was introduced, addressing how a multi-agent model can be positioned in the three-dimensional space defined by the dimensions mentioned; see Figure 1. Relations between models at different abstraction levels were not addressed in [8]. The focus of the current paper is to provide a unified specification format for such interlevel relations. The unified formalisation for interlevel relations introduced here will cover (at least) these abstraction dimensions. The unifying formalisation was inspired by the use of interpretation mappings from logic (e.g., [28]) to describe reduction relations between cognitive and neurological agent models in [22] and [29], and to describe a mapping from a single agent model to a multi-agent model in [11].

The formalisation of interlevel relations introduced here subsumes a number of notions known from the literature. As a first example, for the process abstraction dimension it subsumes not only reduction relations between cognitive and neurological models (e.g., [5], [20], [22], [29]), but also relations between behavioural agent models and cognitive agent models (e.g., [4], [27], [28], [29]). Furthermore, for the temporal abstraction dimension it subsumes the relation between emerging properties of a multi-agent system and the basic mechanisms for the agents (e.g., [1], [2], [3], [6]). Moreover, for the agent cluster dimension it subsumes the relation between agent-based models and population-based models (e.g., [7], [15], [30], [31]) or organisation models (e.g., [11], [14], [18], [23], [26], [32]).

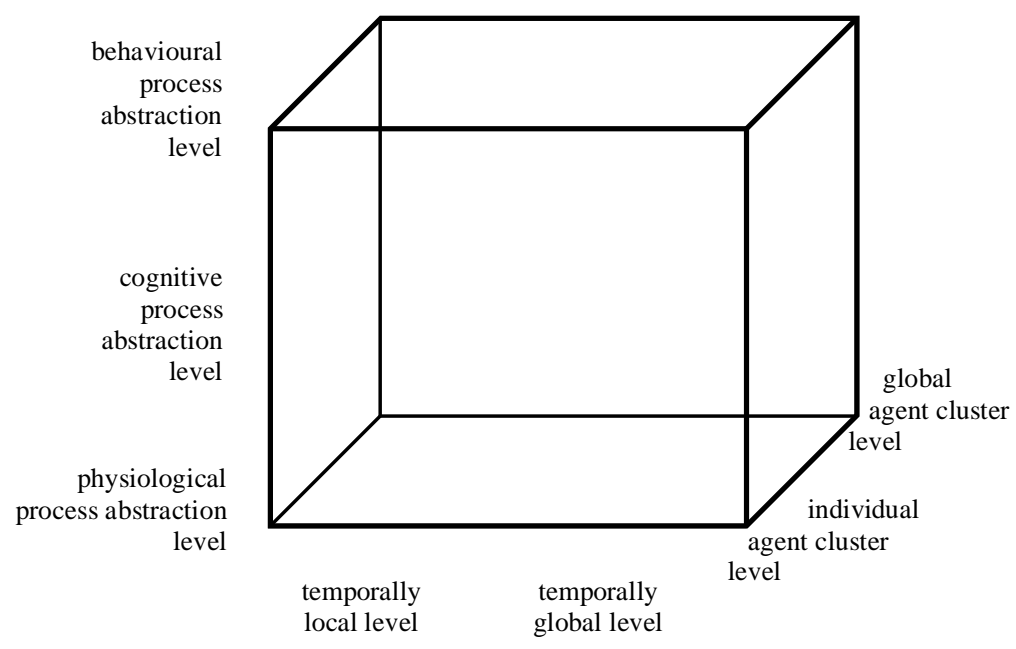

Figure 1. Process abstraction, temporal and agent cluster dimension

The introduced unifying formalisation can play a useful role as a specification format to handle different abstraction levels in analysis and design of multi-agent systems. By making explicit the relationships as part of the specifications, model descriptions (or views) at different levels of abstraction can be freely used while in the meantime keeping track on how they relate. This supports working at an appropriate level of 
abstraction for different subprocesses (e.g., with stakeholders in a more abstract fashion).

The paper is organised as follows. In Section 2 the unified notion of interlevel relation is introduced in a general form. Section 3 addresses how the notion applies to specification of process abstraction interlevel relations, Section 4 of temporal abstraction interlevel relations, and Section 5 of agent cluster abstraction interlevel relations. In Section 6 combination of interlevel relations for different dimensions is discussed. Finally, Section 7 is a discussion.

\section{Specification of Interlevel Relations: Format}

Multi-agent system models are usually specified by temporal relationships (dynamic properties) between states; e.g., [3], [6], [10], but also [1], [15], [19], [25]. Interlevel relations between two models relate the states and dynamic properties specified as part of one model, to states and properties specified as part of the model at the other abstraction level. The introduced general format for specification of interlevel relations between two models involves three key elements:

(1) An ontology mapping to relate basic state properties of the higher level model to state properties of the lower level.

(2) A dynamic property mapping extending the basic ontology mapping to dynamic properties in a (reified) temporal predicate logical language (cf. [16]). This mapping can be applied to dynamic properties that are part of the higher level model, or to dynamic properties that describe patterns in the behaviour of the higher level model.

(3) Logical entailment relations for dynamic properties. Such relationships formally expressed as valid logical implications between temporal predicate logical expressions indicate how mapped higher level properties can be related to properties of the lower level model.

These three key elements are explained in more detail below; see also the overall view depicted in Figure 2. The format was inspired by the use of interpretation mappings from logic to describe reduction relations between cognitive and neurological models in [22]. Within logic (e.g., [28]) an interpretation mapping $\varphi^{*}$ from one theory $T_{2}$ to another one $T_{l}$ usually is defined as

(1) a mapping $\varphi^{*}$ from formulae $F$ in the language of $T_{2}$ to formulae in the language of $T_{1}$,

(2) fulfilling compositionality, and

(3) $\quad T_{2} \vdash F \Rightarrow T_{1} \vdash \varphi^{*}(F)$ for any formula $F$.

The three key elements follow these criteria. 


\subsection{Basic Ontology Mapping}

An interlevel relation for a property expressed as a basic concept (atom) in the ontology used for the level considered, can be defined by means of an ontology mapping of this concept on a concept in the ontology of the other level (criterion (1) of an interpretation mapping). For example, suppose at some level basic state property $b_{2}$ is given: it is warm. Moreover, suppose at a lower level state property $b_{1}$ is given: the molecules have a certain high level of movement. Then the fact that state property $b_{2}$ has an interlevel relation to state property $b_{1}$ can be defined by a basic ontology mapping $\boldsymbol{\varphi}$ :

$$
\varphi\left(b_{2}\right)=b_{1}
$$

When also $b_{1}$ is a basic concept in the ontology of its level, then this $\varphi$ is just a mapping between basic concepts. Note that sometimes also general relations, for example between numbers or time points, are involved that do not belong to any particular level; they are assumed to be mapped onto themselves:

$$
\varphi(\mathrm{T} 1<\mathrm{T} 2)=\mathrm{T} 1<\mathrm{T} 2
$$

It is also possible to define an ontology mapping by mapping basic concepts of one ontology to more complex expressions in the other ontology. For example, suppose $b_{1}$ is the complex property $\exists \vee\left[b_{1}(V) \& \vee \geq 0.3\right]$ where $b_{1}(V)$ is a basic concept at the lower level: the molecules have a level $\mathrm{V}_{1}$ of movement. Then an interlevel relation can be defined as:

$$
\boldsymbol{\varphi}\left(b_{2}\right)=\exists V,\left[b_{1}(V) \quad \& \quad V \geq 0.3\right] .
$$

A basic ontology mapping $\varphi$ can be extended to more complex state properties in a compositional manner, based on rules as:

$$
\begin{aligned}
& \varphi(A \& B)=\varphi(A) \& \varphi(B) \\
& \varphi(A \vee B)=\varphi(A) \vee \varphi(B) \\
& \varphi(A \Rightarrow B)=\varphi(A) \Rightarrow \varphi(B) \\
& \varphi(\neg A)=\neg \varphi(A)
\end{aligned}
$$

\subsection{Dynamic Property Mapping}

For dynamic properties a sorted temporal predicate logic format is assumed with traces and state properties as first class citizens (e.g., [9], [16]). Basic atoms are represented as follows

$$
\begin{array}{ll}
\text { at }(\gamma, \mathrm{T} 1, \mathrm{a}) & \text { in trace } \gamma \text { at time T1 state property a is true } \\
\text { at }(\gamma, \mathrm{T} 2, \neg \mathrm{b}) & \text { in trace } \gamma \text { at time T2 state property b is not true }
\end{array}
$$

When only one fixed trace $\gamma$ is assumed, it can also be simplified to at(T1, a); however, then trace comparison properties (cf. [8], [9]) cannot be expressed. Dynamic 
properties are (sorted) predicate logical expressions built on such atoms, where the state properties a are expressed as terms (reification; cf. [16]). To map dynamic properties a compositionality principle can be used. For example, a dynamic property such as the temporal relation from observation to (temporary) belief, can be mapped by compositionality on the temporal relation from sensor state to sensory representation, when an ontology mapping from state properties observation, resp. belief to sensor state resp. sensory representation is assumed. Suppose that state property $\mathrm{a}_{2}$, resp. $\mathrm{b}_{2}$, at some level has an interlevel relation to state property $\mathrm{a}_{1}$, resp. $b_{1}$, at another level:

$$
\varphi\left(a_{2}\right)=a_{1}, \varphi\left(b_{2}\right)=b_{1},
$$

and a dynamic relation $a_{2} \rightarrow b_{2}$ is given, definable in a temporal predicate logic, for example, by:

$\forall \mathrm{T}[\mathrm{at}(\gamma, \mathrm{T}, \mathrm{a}) \Rightarrow \mathrm{at}(\gamma, \mathrm{T}+\mathrm{D}, \mathrm{b})]$

where $\mathrm{D}$ is a timing parameter (e.g., the step size $\Delta t$ as used in a difference equation format). Then from $a_{2} \rightarrow b_{2}$ the interlevel relations between state properties induce a dynamic property formulated as a temporal relationship $a_{1} \rightarrow b_{1}$. This can be determined by the compositionality principle: the relationships for more complex properties can be determined by the relationships between their components (criterion (2) of an interpretation mapping). Such an interlevel relation is defined by a mapping $\varphi^{*}$ extending the ontology mapping $\varphi$ as follows:

$$
\varphi^{\star}\left(a_{2} \rightarrow b_{2}\right)=\varphi^{\star}\left(a_{2}\right) \rightarrow \varphi^{\star}\left(b_{2}\right) .
$$

Suppose $\varphi\left(\mathrm{a}_{2}\right)=\mathrm{a}_{1}$ and $\varphi\left(\mathrm{b}_{2}\right)=\mathrm{b}_{1}$. Since $\varphi^{*}$ extends $\varphi$, it holds $\varphi^{*}\left(\mathrm{a}_{2}\right)=\varphi\left(\mathrm{a}_{2}\right)$ and $\varphi^{*}\left(\mathrm{~b}_{2}\right)=\boldsymbol{\varphi}\left(\mathrm{b}_{2}\right)$. Therefore $\varphi^{*}$ can be determined for $\mathrm{a}_{2} \rightarrow \mathrm{b}_{2}$ by:

$$
\varphi^{*}\left(a_{2} \rightarrow b_{2}\right)=\varphi^{*}\left(a_{2}\right) \rightarrow \varphi^{*}\left(b_{2}\right)=\varphi\left(a_{2}\right) \rightarrow \varphi\left(b_{2}\right)=a_{1} \rightarrow b_{1} .
$$

When the temporal relationships are depicted graphically, then such an interlevel relationship makes that the graphs are mapped into one another: the mapping preserves shape; see Figure 2. In a similar manner the compositionality principle can be formulated for more complex temporal expressions in a temporal predicate language, by using composition rules such as:

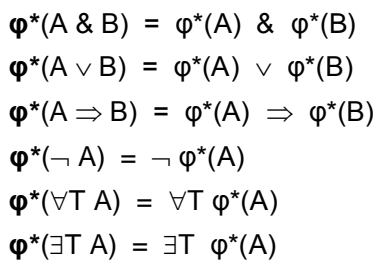


In addition, for basic constituents at $\left(\gamma, \mathrm{T}\right.$, a) of dynamic properties $\varphi^{*}$ is defined as

$\boldsymbol{\varphi}^{*}(\mathrm{at}(\gamma, \mathrm{T}, \mathrm{a}))=\operatorname{at}\left(\boldsymbol{\varphi}^{*}(\gamma), \mathrm{T}, \boldsymbol{\varphi}(\mathrm{a})\right)$

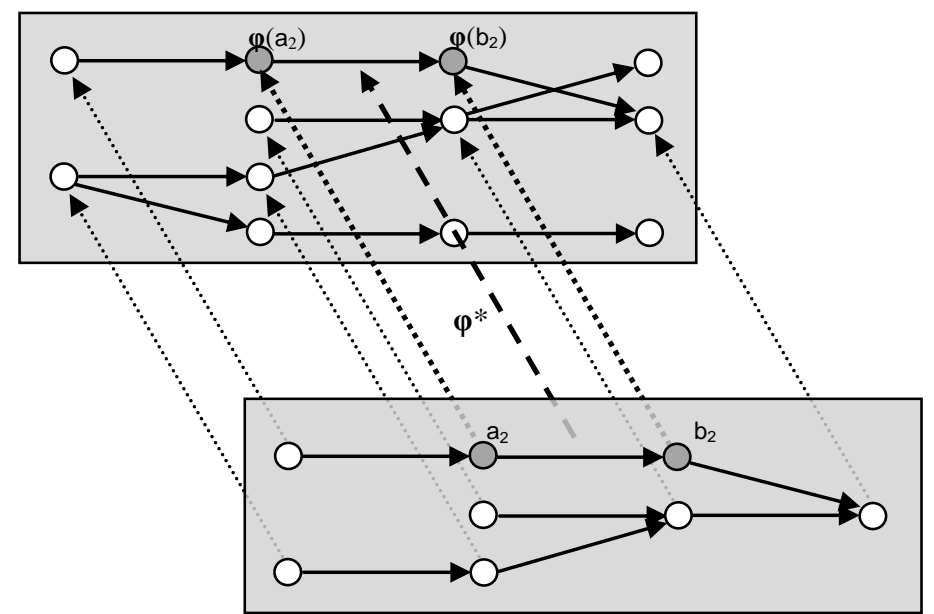

Figure 2. From ontology mapping to dynamic property mapping

where $\varphi^{*}(\gamma)$ is just a name for the mapped trace. Thus a mapping $\varphi^{*}$ is obtained crossing the line between higher level and lower level in Figure 3. For example, using the composition rules, the property

$\forall T 1, T 2[[T 1<T 2 \&$ at $(\gamma, T 1, a) \&$ at $(\gamma, T 2, b)] \Rightarrow \quad \exists T 3$ at $(\gamma, T 3, c)]$

is mapped by $\varphi^{*}$ as follows

$\boldsymbol{\varphi}^{\star}(\forall \mathrm{T} 1, \mathrm{~T} 2[[\mathrm{~T} 1<\mathrm{T} 2 \&$ at $(\gamma, \mathrm{T} 1, \mathrm{a}) \quad \& \quad$ at $(\gamma, \mathrm{T} 2, \mathrm{~b})] \quad \Rightarrow \exists \mathrm{T} 3$ at $(\gamma, \mathrm{T} 3, \mathrm{c})])$

$=\forall \mathrm{T} 1, \mathrm{~T} 2 \quad \varphi^{*}([[\mathrm{~T} 1<\mathrm{T} 2 \&$ at $(\gamma, \mathrm{T} 1, \mathrm{a}) \quad \& \quad$ at $(\gamma, \mathrm{T} 2, \mathrm{~b})] \Rightarrow \exists \mathrm{T} 3$ at $(\gamma, \mathrm{T} 3, \mathrm{c})])$

$=\forall \mathrm{T} 1, \mathrm{~T} 2 \quad\left[\varphi^{*}([\mathrm{~T} 1<\mathrm{T} 2 \&\right.$ at $(\gamma, \mathrm{T} 1, \mathrm{a}) \quad \& \quad$ at $(\gamma, \mathrm{T} 2, \mathrm{~b})]) \Rightarrow \varphi^{*}(\exists \mathrm{T} 3$ at $\left.(\gamma, \mathrm{T} 3, \mathrm{c}))\right]$

$=\forall \mathrm{T} 1, \mathrm{~T} 2 \quad\left[\left[\varphi^{*}(\mathrm{~T} 1<\mathrm{T} 2) \& \varphi^{*}(\mathrm{at}(\gamma, \mathrm{T} 1, \mathrm{a})) \& \varphi^{*}(\mathrm{at}(\gamma, \mathrm{T} 2, \mathrm{~b}))\right] \Rightarrow \exists \mathrm{T} 3 \quad \varphi^{*}(\mathrm{at}(\gamma, \mathrm{T} 3, \mathrm{c}))\right]$

$=\forall \mathrm{T} 1, \mathrm{~T} 2\left[\left[\mathrm{~T} 1<\mathrm{T} 2 \& \operatorname{at}\left(\varphi^{*}(\gamma), \mathrm{T} 1, \boldsymbol{\varphi}(\mathrm{a})\right) \& \operatorname{at}\left(\varphi^{*}(\gamma), \mathrm{T} 2, \boldsymbol{\varphi}(\mathrm{b})\right)\right] \Rightarrow \exists \mathrm{T} 3\right.$ at $\left.\left(\varphi^{*}(\gamma), \mathrm{T} 3, \boldsymbol{\varphi}(\mathrm{c})\right)\right]$

\subsection{Logical Relationships between Properties}

Although cases such as the one depicted in Figure 2 may suggest differently, usually a mapped higher level model is not exactly equal to a given lower level model, but instead, according to criterion (3) of an interpretation mapping it can be logically related to it by a logical entailment relation between dynamic properties. As an example, suppose an ontology mapping is given with

$$
\boldsymbol{\varphi}\left(\mathrm{a}_{2}\right)=\mathrm{a}_{1} \text { and } \boldsymbol{\varphi}\left(\mathrm{d}_{2}\right)=\mathrm{d}_{1}
$$

and as part of the higher level model a temporal relationship $a_{2} \rightarrow d_{2}$ is specified. Moreover, suppose in the lower level model the relationships 


$$
a_{1} \rightarrow b_{1}, b_{1} \rightarrow c_{1}, c_{1} \rightarrow d_{1}
$$

are specified, then an interlevel relation may relate $a_{2} \rightarrow d_{2}$ to the conjunction

$$
a_{1} \rightarrow b_{1} \& b_{1} \rightarrow c_{1} \& c_{1} \rightarrow d_{1}
$$

However, as by the ontology mapping $\varphi$ the basic state property $a_{2}$ is mapped on $a_{1}$, and $d_{2}$ on $d_{1}$, by compositionality $a_{2} \rightarrow d_{2}$ is mapped to the lower level dynamic property $a_{1} \rightarrow d_{1}$, i.e.,

$$
\varphi^{*}\left(a_{2} \rightarrow d_{2}\right)=\varphi^{*}\left(a_{2}\right) \rightarrow \varphi^{*}\left(d_{2}\right)=a_{1} \rightarrow d_{1}
$$

The latter lower level dynamic property is not part of the lower level model itself, but at least can be logically related to such dynamic properties by the logical entailment relation specified as a valid logical implication (with appropriate timing parameters):

$$
a_{1} \rightarrow b_{1} \& b_{1} \rightarrow c_{1} \& c_{1} \rightarrow d_{1} \Rightarrow a_{1} \rightarrow d_{1}
$$

So in this case the interlevel relation between the dynamic property $a_{2} \rightarrow d_{2}$ on the one hand and the three dynamic properties

$$
a_{1} \rightarrow b_{1}, b_{1} \rightarrow c_{1}, c_{1} \rightarrow d_{1}
$$

on the other hand, can be formulated as a combination of compositionality and a logical relationship that can be specified as a valid logical implication.

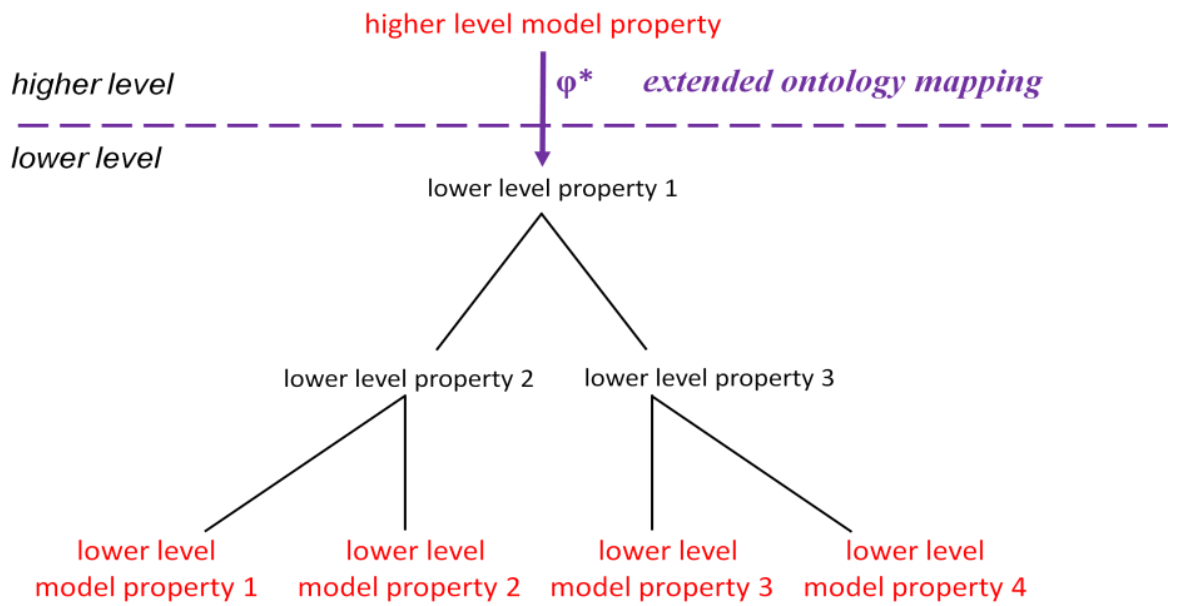

Figure 3. Unified format of an interlevel relation specification 
Such a logical implication for multiple properties can be graphically represented in a hierarchical manner in a more general form as depicted in Figure 3 under the line between higher and lower level. In the subsequent three sections it will be shown how this general notion of interlevel relation can be applied for the three different types of abstraction dimensions considered: for the process abstraction (using extended ontology mapping $\pi^{*}$ ), temporal abstraction (using $\theta^{*}$ ), and agent cluster abstraction (using $\rho^{*}$ ) dimension; see Figure 4.

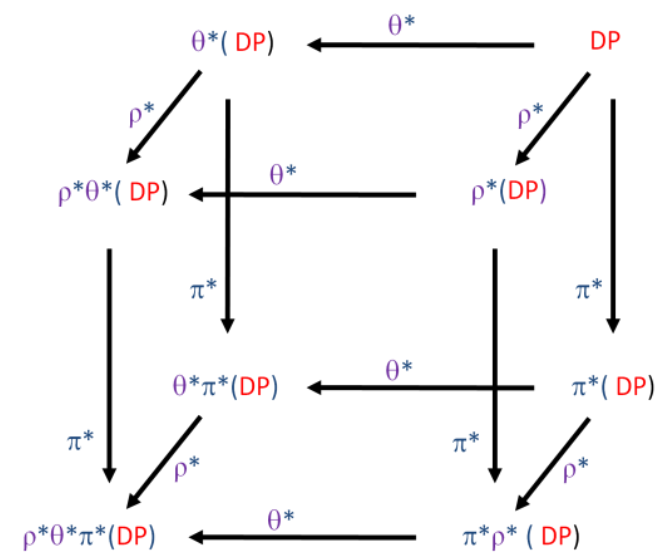

Figure 4. Overview of interlevel relations for three dimensions

\section{Interlevel Relations for the Process Abstraction Dimension}

For the process abstraction dimension, agent processes can be conceptualised from an external behavioural perspective (e.g., [4], [27]) or from an internal cognitive or neurological perspective (e.g., [8], [22], [29]). In this section interlevel relations are discussed between a behavioural, cognitive, and neurological level model for a given process in reality. In [8] an instant Internet dating context was used as a toy example to illustrate different abstraction levels, around an Internet agent ID that can be contacted when somebody wants a date. This is to be considered a fictitious example just for the purpose of illustration. In principle many activities during a date are possible, such as hiking, going out for a dance club, attending a concert, et cetera. For the case study three characteristics make up a type of an activity: active or not, involving eating or not, and romantic or not. Moreover, it is assumed that dating is steered by an identity described by specific body states: blood sugar level, adrenaline level, testosterone level, and dopamine lack. Each person involved has interaction with the Internet agent ID in three phases:

- Based on a chosen identity the person requests ID for a date

- After a candidate has been proposed by ID the person proposes ID a desired activity type

- When an activity type confirmed by a candidate is desired, a date with this candidate is performed 
In this example at the behavioural level a person $\mathrm{P}$ (with respect to the Internet dating service agent ID) is described as an agent by three behavioural properties BP1 to BP3, respectively, for generating a date request to ID (based on observed dopamine lack), proposing an activity (when a candidate is offered by ID), and performing the date (when ID communicates that the candidate agrees). To keep the example a bit simple the detailed matchmaking aspects to pair date requests by ID (based on the persons' profiles) have been left out of consideration. For this example the ontology used at the behavioural level makes use of atoms such as

observes $(P, a)$

communication(P1, a, P2)

performs $(\mathrm{P}, \mathrm{b})$

where $\mathrm{a}$ is a world state property and $\mathrm{b}$ is an action, and P, P1, P2 are agents (i.e., persons or the agent ID). The behavioural properties BP1, BP2, BP3 describe a person $P$ 's process to come to a date. Here $\operatorname{act}(X)$ denotes an activity that fits adrenalin level $X$ (i.e., the higher $X$, the more active the activity).

\section{BP1 Requesting for a date}

If $P$ observes that his or her dopamine lack is high,

then $P$ will request for a date

$\forall \mathrm{T}, \mathrm{P} \quad[[\mathrm{at}(\gamma, \mathrm{T}, \operatorname{observes}(\mathrm{P}, \operatorname{body}($ dopaminelack, high $)))$

$\Rightarrow \exists T 1 \quad[\mathrm{~T} 1 \geq \mathrm{T} \&$ at $(\gamma, \mathrm{T} 1, \mathrm{communication}(\mathrm{P}$, date_request, ID)) ]

\section{BP2 Proposing an activity}

If it was communicated to $P$ that $Q$ agrees in a date,

and $P$ observes that his or her adrenaline level is $X$

then $P$ will propose to perform activity $\operatorname{act}(X)$.

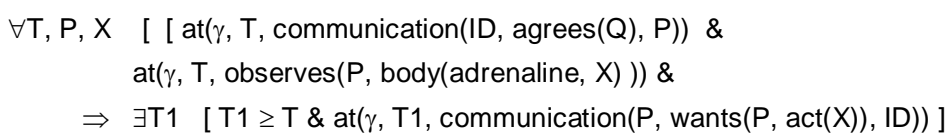

\section{BP3 Performing an agreed activity}

If it was communicated to $P$ that $Q$ agrees in having a date

and $P$ observes adrenaline level $X$

and it was communicated to $P$ that $Q$ wants to do activity $\operatorname{act}(X)$,

then $P$ will perform $\operatorname{act}(X)$ with $Q$.

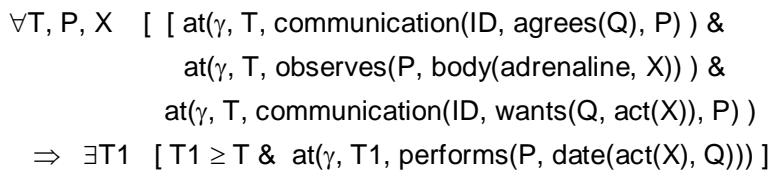

At the cognitive level the ontology used is based on atoms

$\operatorname{sensing}(P, a)$, belief $(P, a)$, desire $(P, b)$, intention $(P, b)$, effector $(P, b)$, 
where sensing refers to the same state as observes and effector to the same state as performs.

\section{From Behavioural to Cognitive Level}

The ontology mapping $\pi$ of basic behavioural state properties onto cognitive state properties is defined by

. $\pi(\operatorname{observes}(P, a))=\operatorname{sensing}(P . a) \quad \pi(\operatorname{performs}(P, b))=\operatorname{effector}(P . b)$

The extension $\pi^{*}$ of this mapping to dynamic properties is defined by compositionality. What remains is how a mapped behavioural dynamic property relates to (multiple) dynamic properties from the cognitive model. A mapped behavioural dynamic property may be considered as a kind of shortcut sensing $\rightarrow$ effector of a chain involving internal cognitive states; for example:

$$
\text { sensing } \rightarrow \text { belief } \rightarrow \text { desire } \rightarrow \text { intention } \rightarrow \text { effector }
$$

In a logical sense such a shortcut can be modelled as a valid logical implication from the properties describing the successive steps to the property expressing the shortcut:

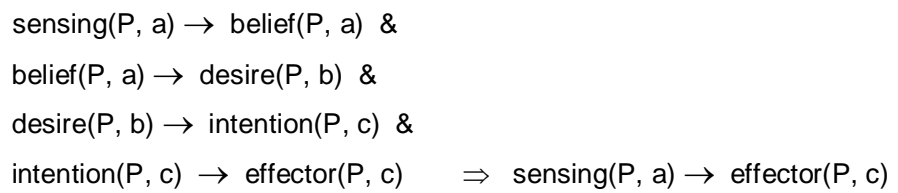

For the Internet dating example at the cognitive level a number of dynamic properties relate relevant beliefs, desires and intentions. For example, the following simplified properties describe the cognitive process to generate a date request.

\section{LCP1 Generating a desire to date}

If $P$ senses that her or his dopamine lack is high

then $P$ will have the desire to obtain a date

$\forall T, P$ at $(\gamma, T$, sensing $(P, \operatorname{body}($ dopaminelack, high $))) \Rightarrow \exists T 1 \geq T$ at $(\gamma, T 1, \operatorname{desire}(P$, date $))$

LCP2 Generating an intention to request a date based on a desire

If $P$ has the desire to obtain a date,

then $P$ will have the intention to request a date.

$\forall \mathrm{T}, \mathrm{P}$ at $(\gamma, \mathrm{T}, \operatorname{desire}(\mathrm{P}$, date $) \Rightarrow \exists \mathrm{T} 1 \geq \mathrm{T}$ at $(\gamma, \mathrm{T} 1$, intention( $\mathrm{P}$, date_request $))$

\section{LCP3 Requesting for a date based on the intention}

If $P$ has an intention to request a date,

then $P$ will request $I D$ for a date

$\forall T, P$ at $(\gamma, T$, intention( $P$, date_request $) \Rightarrow$

$\exists T 1 \geq T$ at $(\gamma, T 1$, communication( $P$, date_request, ID $))$

The other cognitive properties LCP4 to LCP10 are similar. For this case the following entailment relations specified as valid implications can be used to define the interlevel relation (see Figure 5). 


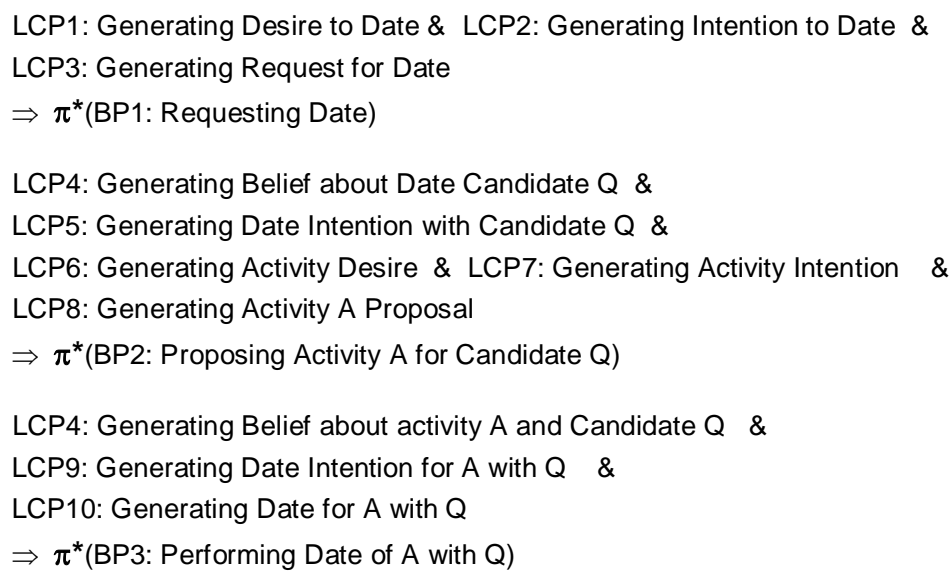

More examples of interlevel relations for this process abstraction dimension, in particular, for the relation between cognitive and neurological level models can be found in [22] and [28].
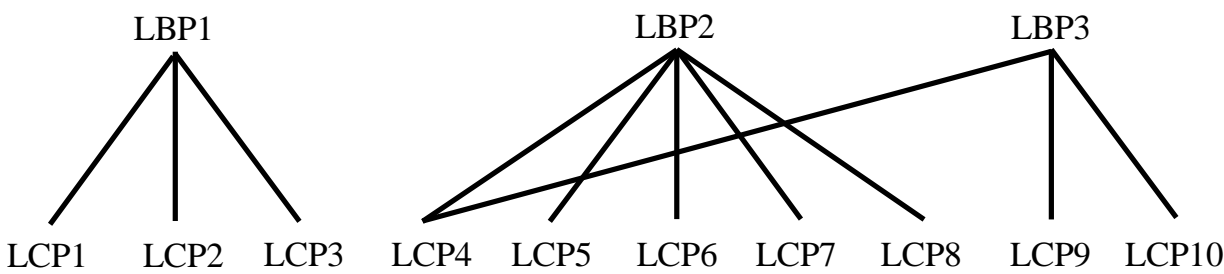

Figure 5. Valid implications as a key element of an interlevel relation specification: relating behavioural properties to properties of the cognitive model

\section{From Cognitive to Physiological Level}

The next question addressed for the ID case study is: how are cognitive state properties and dynamic properties related to physiological state properties and dynamic properties. In accordance with the elements discussed above, the following steps are made:

1. Define interlevel relations for cognitive state properties based on an ontology mapping.

2. Extend this ontology mapping by compositionality to all dynamic properties in the cognitive model.

3. Consider how the mapped dynamic properties can be related to the dynamic properties defining the physiological model. 
First it is addressed how cognitive state properties (and input and output state properties) can be mapped onto physiological state properties. Cognitive state properties concern different types of beliefs and desires and intentions, and input and output such as observations, communications and actions. Table 1 shows how they can be mapped onto physiological state properties. Here one of the main issues is that the activation level of neuronal state is mapped onto a binary all-or-nothing format used for the cognitive model, where for activation level below 0.5 the presence of the state property is considered low and not to occur and from 0.5 on it is considered high and to occur. Note that for the cognitive model beliefs are considered not to persist long, and therefore can be related to sensory representation activations. For beliefs that are meant to be detached from observation and persist over longer time periods, this option cannot be used, as neuronal activations do not persist over longer time periods. In such a case an option would be to relate them to strengths of connections between neurons, that are modified by Hebbian learning.

Moreover, for this case desires are assumed to express body states that deviate from what they should be from the viewpoint of homeostasis of the organism. Therefore they are just related to body maps represented by the sensory representations of body states. For example, a high adrenaline level is related to a desire of activities that make use of this, i.e., more active activities. Similarly, a low blood sugar level is related to the desire for activities that compensate this, e.g., involving eating.

\begin{tabular}{|c|c|}
\hline Cognitive state property & Physiological state property \\
\hline observed(P, B, low) & $\exists \mathrm{V}$ activation $(\mathrm{S}(\mathrm{P}, \mathrm{B}), \mathrm{V}) \& \mathrm{~V}<0.5$ \\
\hline observed( $P, B$, high) & $\exists \mathrm{V}$ activation $(\mathrm{S}(\mathrm{P}, \mathrm{B}), \mathrm{V}) \& \mathrm{~V} \geq 0.5$ \\
\hline communication $(X, I D, P)$ & $\exists \mathrm{V}$ activation $(S(P, X), V) \& V \geq 0.5$ \\
\hline belief $(P, X)$ & $\exists \mathrm{V}$ activation $(\mathrm{SN}(\mathrm{P}, \mathrm{X}), \mathrm{V}) \& \mathrm{~V} \geq 0.5$ \\
\hline desire $(P$, activity_for $(B, X))$ & $\begin{aligned} \exists V \quad[\text { activation }(S N(P, B), V) \& \\
\quad[(X=\text { high \& } V \geq 0.5) \vee(X=\text { low \& } V<0.5)]\end{aligned}$ \\
\hline intention $(P, X)$ & $\exists \mathrm{V}$ activation $(\mathrm{PN}(\mathrm{P}, \mathrm{X}), \mathrm{V}) \& \mathrm{~V} \geq 0.5$ \\
\hline communication $(\mathrm{X}, \mathrm{P}, \mathrm{ID})$ & $\exists V$ activation $(E(P, X), V) \& V \geq 0.5$ \\
\hline performed $(P, X)$ & $\exists V$ activation $(E(P, X), V) \& V \geq 0.5$ \\
\hline
\end{tabular}

Table 1. Examples of the ontology mapping $\pi_{\mathrm{ID}}$ from cognitive to physiological level

Intentions are considered as preparations for actions, as also modelled by preparation neuron activations. Therefore they are mapped onto these neuron activations. Finally, observations and incoming communications are related to sensor states, and action performance and outgoing communications to effector states. More details about interlevel relations between cognitive and physiological level can be found in [29]. 


\section{Interlevel Relations for the Temporal Dimension}

Temporally local descriptions specify the steps made by the basic mechanisms of a process, whereas temporally more global descriptions describe patterns that result or emerge from these basic mechanisms. Such temporally global properties are often used to express (behavioural) requirements on a model, and can be used in formal verification (e.g., [9], [12], [14]). Temporal interlevel relations can be viewed as a description of to which temporally local descriptions such emerging patterns relate, as also is addressed in compositional verification and model checking; see for example, [2], [6], [9], [12], [17], [26]. Patterns emerging over time can be of many different types, for example varying from the last drop that makes the bucket flow over, to monotonicity relations within one trace or between different traces, and equilibria.

Temporal interlevel relations can be defined within any process abstraction level from neurological to cognitive or behavioural. Depending on the type of emerging property that is considered, temporal interlevel relations may involve logical and/or mathematical elements. Also in the temporal dimension, at different levels different ontologies may be used. Therefore in general temporal interlevel relations are specified by (1) using an ontology mapping $\theta$ from the temporally higher level ontology to the temporally lower level ontology, (2) compositionally extending $\theta$ to $\theta *$ for more complex expressions, and (3) logically relating a mapped property to a number of properties from the lower level model. As an example, consider to determine the overall number of drops falling into a bucket over time to find out that the bucket will flow over at some point in time. Suppose, to make it less trivial, the tap filling a bucket is opened more and more over time, in a linear fashion, described by the following local property: at each time point $t$ (in seconds) the number of drops added per second is $a t$. Moreover, suppose a global property is formulated: at time $T$ the bucket contains $K$ drops of water. Then the global property can be related to the local property using the following mathematical relation:

$$
\sum_{\mathrm{t}=0}^{\mathrm{T}} \operatorname{drops}(\mathrm{t})=\mathrm{K}
$$

This can be rewritten as:

$$
\sum_{\mathrm{t}=0}^{\mathrm{T}} \text { at }=\mathrm{K} \quad \Leftrightarrow 1 / 2 \text { a } \mathrm{T}(\mathrm{T}+1)=\mathrm{K} .
$$

This example shows how a temporal interlevel relation can be obtained by (mathematical) integration of the small steps in the process over time to obtain their overall effect after a longer time period.

As in Section 3, temporal interlevel relations will be illustrated for the dating case study. An example of a temporal interlevel relation at the behavioural process abstraction level will be discussed, in particular, concerning a body state that is achieved. 


\section{GBP1 Body state achieved with low dopamine lack}

If at some point in time $\mathrm{T}$ the dopamine lack is high

then at a later time point $\mathrm{T} 1$ the dopamine lack will be low.

$\forall \mathrm{T}, \mathrm{P}, \mathrm{V}[\mathrm{at}(\gamma, \mathrm{T}, \operatorname{body}(\mathrm{P}, \operatorname{dopaminelack}$, high $)) \Rightarrow$

$\exists T 1, V 1[T 1 \geq T \&$ at $(\gamma, T 1, \operatorname{body}(P$, dopaminelack, low $)]$

Note that this property is formulated in terms of an ontology for the temporally global level (which is taken qualitative). The ontology mapping $\boldsymbol{\theta}$ maps the temporally global atoms on atoms (taken quantitative) at the local level as follows:

$\theta(\operatorname{at}(\gamma, T$, body $(P$, dopaminelack, high $))=\exists \mathrm{V}$ at $(\gamma, T 1$, body_state $(P$, dopaminelack, $V)) \& V \geq 0.5$

$\theta(\operatorname{at}(\gamma, T$, body $(P, \operatorname{dopaminelack,~low~}))=\exists \mathrm{V}$ at $(\gamma, T 1$, body_state $(P$, dopaminelack, $V)) \& V<0.5$

Dynamic property GBP1 relates to behavioural dynamic properties BP1, BP2, BP3; BBP1, BPP2 at the temporally local level describing an interaction between the behavioural processes of the person and the dynamics of the body state. The first three were described already in Section 3; the last two are as follows:

\section{BBP1 Generating a body state observation}

If $\quad$ in $P$ body state property $B$ has strength $V<0.5$

then it will be observed by $P$ that $B$ is low.

$\forall \mathrm{T}, \mathrm{P}, \mathrm{V}[[$ at $(\gamma, \mathrm{T}$, body_state $(\mathrm{P}, \mathrm{B}, \mathrm{V})) \& \mathrm{~V}<0.5] \Rightarrow$

$\exists \mathrm{T} 1 \quad[\mathrm{~T} 1 \geq \mathrm{T} \&$ at $(\gamma, \mathrm{T} 1, \operatorname{observes}(\mathrm{P}, \operatorname{body}(\mathrm{B}$, low $)))$

If $\quad$ in $P$ body state property $B$ occurs of strength $V \geq 0.5$

then it will be observed by $P$ that $B$ is high.

$\forall T, P, V \quad[\quad[$ at $(\gamma, T$, body_state $(P, B, V)) \& V \geq 0.5] \Rightarrow$

$\exists T 1 \quad[T 1 \geq T \&$ at $(\gamma, T 1, \operatorname{observes}(P, \operatorname{body}(B, h i g h)))$

\section{BBP2 From a date to its effect}

If at $T$ a date with $Q$ doing an activity of type $X$ is performed

and the level of the body state for adrenaline qualifies as $X$

then at a later time point $T l$ within time duration $D$ after $T$ the body states for adrenaline and dopamine lack will have levels $<0.5$.

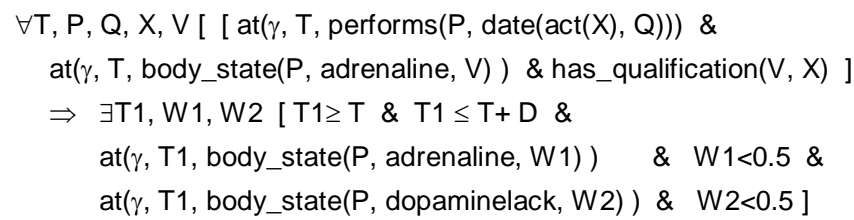

These behavioural properties together entail the global property GBP1:

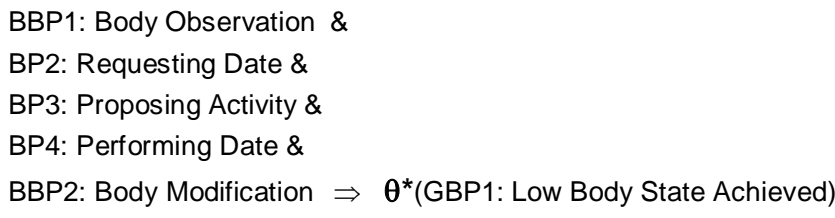

Thus a temporal interlevel relation is obtained. 
Notice that temporal interlevel relations themselves from the cognitive level can be mapped by $\pi_{\mathrm{ID}} *$ onto interlevel relations at the physiological level. For example, the cognitive achievement property GCP1 can be mapped by $\pi_{\mathrm{ID}} *$ to a physiological achievement property GPP1:

$$
\pi_{\mathrm{ID}} *(\mathrm{GCP} 1)=\mathrm{GPP} 1
$$

This mapping extends to the whole tree representing a temporal interlevel relation for GCP1 and provides an interlevel relation for GPP1. Within such an approach two principles for mapping interlevel relations play a role:

Commutation principle (see Figure 6)

$$
\pi^{*}\left(\theta^{*}(\mathrm{DP})\right)=\theta^{*}\left(\pi^{*}(\mathrm{DP})\right)
$$

Compositionality principle

$$
\begin{aligned}
& \pi^{*}(\mathrm{~A} \& \mathrm{~B} \Rightarrow \mathrm{C})=\pi^{*}(\mathrm{~A}) \& \pi^{*}(\mathrm{~B}) \Rightarrow \pi^{*}(\mathrm{C}) \\
& \theta^{*}(\mathrm{~A} \& \mathrm{~B} \Rightarrow \mathrm{C})=\theta^{*}(\mathrm{~A}) \& \theta^{*}(\mathrm{~B}) \Rightarrow \theta^{*}(\mathrm{C})
\end{aligned}
$$

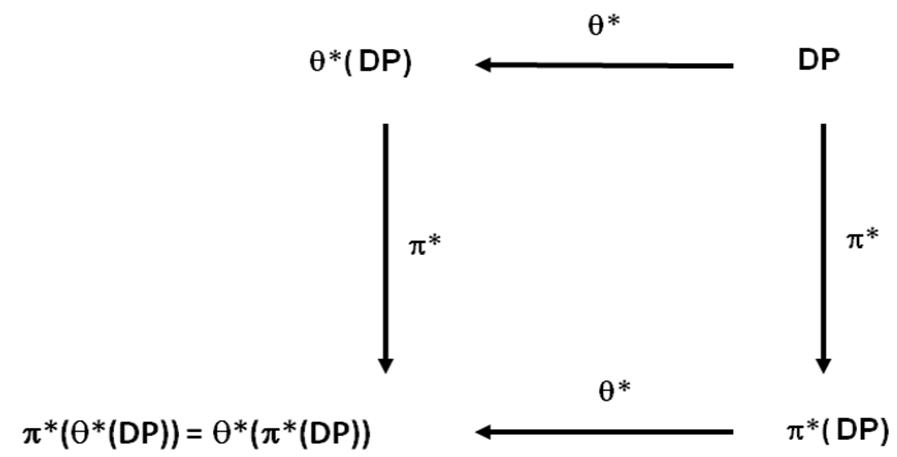

Figure 6. Commutation principle for extended ontology mappings

As this is symmetric, there are in fact two ways to map interlevel relations:

Using $\pi^{*}$

From a temporal interlevel relation for higher process abstraction level to temporal interlevel relation for lower process abstraction level. E.g., from cognitive temporal interlevel relation to physiological temporal interlevel relation.

$U \operatorname{sing} \theta^{*}$

From a process abstraction interlevel relation at higher temporal level to process abstraction interlevel relation at lower temporal level. E.g., from temporally global process abstraction interlevel relation to temporally local process abstraction interlevel relation. 


\section{Interlevel Relations for the Agent Cluster Dimension}

In the third dimension considered a population, group or cluster of agents can be abstracted to one agent. For example, in the extreme case the whole population of individuals can be considered as one super-agent. An example of such a perspective is the Gaia hypothesis which considers the earth as a whole as one intelligent organism (cf. [21]). As another example, in [11] it was addressed how an ant society which uses pheromones on the ground as a form of shared extended mind can be interpreted as a single agent by using a mapping from a single agent model onto the multi-agent model of the ant society. Other literature which relates a single agent perspective to multiple agents can be found in [23] where the mind is considered to emerge as an interaction of a large number of agents within the brain, and in [7], [14], [18], [32].

Models at different levels of the agent cluster dimension describe a process in reality according to different grain-sizes of the basic entities modelled as agents. Each of the agents used in a higher level model refers to an element in reality that is modelled as a group or cluster of agents in a lower level model. Given this, in an agent cluster interlevel relation, to relate two models at different agent cluster levels, each agent in the higher level model is related to a cluster of agents in the lower level model. Moreover, states and properties of a higher level agent are related to states and properties of individual agents in the set (cluster) of lower level agents that relates to this higher level agent. In this section by a few examples it will be discussed how an agent cluster interlevel relation can be defined for behavioural models and for cognitive models. First a basic ontology mapping $\rho$ is defined. Properties from the higher cluster level are collective properties that often refer to an aggregated number for the size or strength of a certain cluster or property thereof. The general idea is depicted in Figure 7.

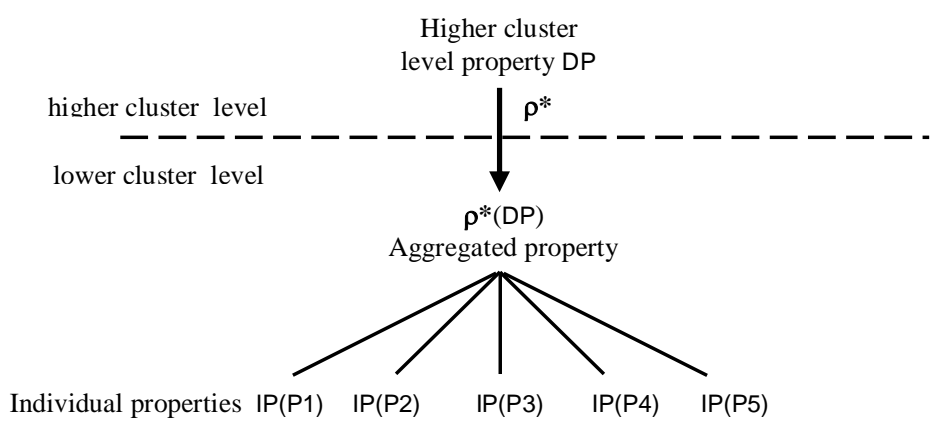

Figure 7. Aggregation of individual agent properties 
The extended ontology mapping $\rho^{*}$ maps a cluster property DP onto the aggregated properrty $\rho^{*}(\mathrm{DP})$, and this aggregated property is implied by a conjunction of the individual properties $\mathrm{IP}(\mathrm{P})$ for the different individuals $\mathrm{P} 1, \ldots, \mathrm{P}$.

To define within the lower level an aggregated property two issues need to be addressed:

(i) a language element is needed to represent an accumulation over a group of agents

(ii) the clusters and the agents belonging to them may be dynamic, and in the lower level ontology no state properties may be available to indicate explicitly which agents belong to which cluster at some point in time

For the issue (i), this can be solved in a sorted temporal predicate logical language by introducing the following abbreviation for summation of values V1 or V2, depending on the truth of a formula $\varphi$ containg a free variable $\mathrm{X}$ over a sort $\mathrm{S}$ :

$\Sigma_{\mathrm{X}: \mathrm{S}} \operatorname{case}(\psi, \mathrm{V} 1, \mathrm{~V} 2)=\mathrm{V}$.

Here for any (temporal) formula $\psi$, the expression $\operatorname{case}(\psi, \mathrm{V} 1, \mathrm{~V} 2)$ indicates the value $\mathrm{V} 1$ if $\psi$ is true, and V2 otherwise. In particular, when $\mathrm{V} 1=1$ and $\mathrm{V} 2=0$ is chosen, this represents the number of elements $\mathrm{X}$ within sort $\mathrm{S}$ for which $\psi$ is true. This construct can be considered logically as a short notation for a large disjunction of conjunctions over many instances. It will be applied to define the basic ontology mapping from the higher to the lower cluster level, with $\mathrm{S}$ the sort of all agents and $\mathrm{P}$ in $\psi$ the variable $\mathrm{X}$. For this instantiation of $\mathrm{S}$ the following naming will be used:

agg_number_of_agents_with $(\mathrm{V}, \psi) \equiv \Sigma_{\mathrm{P}: \mathrm{S}} \operatorname{case}(\psi, 1,0)=\mathrm{V}$

Due to issue (ii), usually it is not possible to simply relate state properties of the higher level to state properties at the lower level. However, it is still possible to map the temporal atoms of the form at $(\gamma, \mathrm{T}$, a) with a higher level state property a onto dynamic properties at the lower level. For the Internet dating case, as an illustration the following (dynamic) clusters are considered:

- persons in the process of obtaining a date: those who requested a date but did not yet start to perform a date $(R)$

- persons performing a date (D)

- persons not in a process of dating: not in a date nor in a process of obtaining a date $(\mathrm{N})$; this is the rest of the population

\section{Behavioural Cluster Interlevel Relations}

To apply the solutions of issues (1) and (2) at the behavioural level, as a first example, consider the state property has_size(D, V). Whether at some point in time $T$ 
an agent belongs to this group $D$ can be expressed in a formula $\psi_{D}$ as a dynamic property as follows.

$\psi_{\mathrm{D}}(\gamma, \mathrm{T}, \mathrm{P}) \equiv \exists \mathrm{A}, \mathrm{Q}$ at $\left.(\gamma, \mathrm{T}, \operatorname{performs}(\mathrm{P}, \operatorname{date}(\mathrm{A}, \mathrm{Q})))\right)$

So, this is a case for which the membership of the cluster can be characterised by a lower level state property: performs $(P, \operatorname{date}(A, Q))$. Note that this formula contains trace $\gamma$, agent $P$ and time point $T$ as a free variable. This formula $\psi_{D}$ is used to define the ontology mapping $\rho$ as follows:

$\boldsymbol{\rho}(\mathrm{at}(\gamma, \mathrm{T}$, has_size $(\mathrm{D}, \mathrm{V}))))=$ agg_number_of_agents_with $\left(\mathrm{V}, \psi_{\mathrm{D}}\right)$

For the sake of simplicity here the names of the traces $\gamma$ at the higher and lower level are taken identical.

Next, consider the state property has_size( $R, V)$. For this group $R$ all individuals are taken into account who did communicate a request for a date until the current point in time, but did not yet start a date. Whether at some point in time $T$ an agent belongs to group $\mathrm{R}$ is expressed as a dynamic property $\psi_{\mathrm{R}}$ at the lower cluster level as follows (note there is no state property available this time).

$$
\begin{gathered}
\psi_{R}(P, T) \equiv \exists T 1[T 1 \leq T \quad \& \text { at }(\gamma, T 1, \operatorname{communication}(P, \text { date_request, ID }))) \\
\& \neg \exists T 2, A, Q[T 1 \leq T 2 \leq T \text { \& at }(\gamma, T 2, \operatorname{performs}(P, \operatorname{date}(A, Q))))]]
\end{gathered}
$$

This formula $\psi_{\mathrm{R}}$ can be used to define:

$$
\rho(a t(\gamma, T \text {, has_size }(R, V)))=\text { agg_number_of_agents_with }\left(\mathrm{V}, \psi_{\mathrm{R}}\right)
$$

Finally, for the agents related to cluster $\mathrm{N}$ it can be expressed that they are all agents

\begin{tabular}{|c|c|}
\hline Global cluster level property & Local cluster level property \\
\hline $\operatorname{state}(\gamma, T) \mid=$ has_size $(D, V)$ & $\begin{array}{l}\text { is_aggregated_number_of_agents_with }\left(V, \varphi_{D}\right) \\
\text { with } \\
\left.\left.\varphi_{D}=\exists A, Q \text { state }(\gamma, T) \mid=\text { performed }(P, \operatorname{date}(A, Q))\right]\right]\end{array}$ \\
\hline $\operatorname{state}(\gamma, T) \mid=$ has_size $(R, V)$ & $\begin{array}{l}\text { is_aggregated_number_of_agents_with }\left(V, \varphi_{R}\right) \\
\text { with } \\
\varphi_{R}=\exists T 1, I[T 1 \leq T \text { \& } \\
\text { state }(\gamma, T 1) \mid=\text { communication }(P, \text { date_with_id }(I), I D) \& \\
\neg \exists T 2, A, Q[T 1 \leq T 2 \leq T \text { \& state }(\gamma, T 2) \mid=\operatorname{performed}(P, \operatorname{date}(A, Q))]]\end{array}$ \\
\hline $\operatorname{state}(\gamma, \mathrm{T}) \mid=$ has_size $(\mathrm{N}, \mathrm{V})$ & is_aggregated_number_of_agents_with $\left(\mathrm{V}, \neg \varphi_{R} \& \neg \varphi_{D}\right)$ \\
\hline $\operatorname{state}(\gamma, T) \mid=$ has_size $(\mathrm{N}(B), V)$ & $\begin{array}{l}\text { is_aggregated_number_of_agents_with }\left(\mathrm{V}, \varphi_{\mathrm{N}, \mathrm{B}}\right) \\
\quad \text { with } \\
\begin{aligned} \varphi_{\mathrm{N}, \mathrm{B}}=\neg & \varphi_{\mathrm{R}} \& \neg \varphi_{\mathrm{D}} \& \\
& \quad \exists \mathrm{V} 1[\operatorname{state}(\gamma, \mathrm{T}) \mid=\text { body_state }(\mathrm{P}, \mathrm{B}, \mathrm{V} 1) \& \mathrm{~V} 1 \geq 0.5])\end{aligned}\end{array}$ \\
\hline
\end{tabular}
not in R nor in $D$ : $\psi_{N}=\neg \psi_{R} \& \neg \psi_{D}$.

$$
\rho\left(\operatorname{at}\left(\gamma, \mathrm{T}, \mathrm{has} \_\operatorname{size}(\mathrm{N}, \mathrm{V})\right)\right)=\text { agg_number_of_agents_with }\left(\mathrm{V}, \psi_{\mathrm{N}}\right)
$$

Table 2. Ontology mapping $\rho_{\text {ID }}$ from global to local cluster level: behavioural process abstraction level 
This ontology mapping $\rho$ can be extended by compositionality to a mapping $\rho *$ for dynamic properties. As an example, the following dynamic property LCBP1 is taken; here $\mathrm{N}(\mathrm{do})$ denotes the subgroup of $\mathrm{N}$ with high dopamin lack.

\section{LCBP1 Dynamics of group $\mathbf{N}$}

If $\quad N$ has size $V$,

and the part $N(d o)$ of $N$ with dopamine lack level high has size $V l$,

and $D$ has size $V 2$,

then at some later point in time $N$ will have size $V+(\alpha V 2-\beta V 1) \Delta t$

at $(\gamma, T$, has_size $(\mathrm{N}, \mathrm{V})) \&$ at $(\gamma, \mathrm{T}$, has_size $(\mathrm{N}(\mathrm{do}), \mathrm{V} 1)) \&$ at $(\gamma, \mathrm{T}$, has_size $(\mathrm{D}, \mathrm{V} 2))$

$\Rightarrow \exists T 1[T 1 \geq T \&$ at $(\gamma, T 1$, has_size $(N, V+(\alpha \mathrm{V} 2-\beta V 1) \Delta t))]$

Here $\alpha$ is the fraction per time unit in $D$ finishing a date, and $\beta$ is the fraction per time unit in $\mathrm{N}$ requesting a date. Taking $\psi_{\mathrm{N}(\mathrm{do})}(\gamma, \mathrm{T}, \mathrm{P})=\psi_{\mathrm{N}} \quad \& \quad \operatorname{at}(\gamma, \mathrm{T}$, body $(\mathrm{P}$, dopaminelack, high)), it holds:

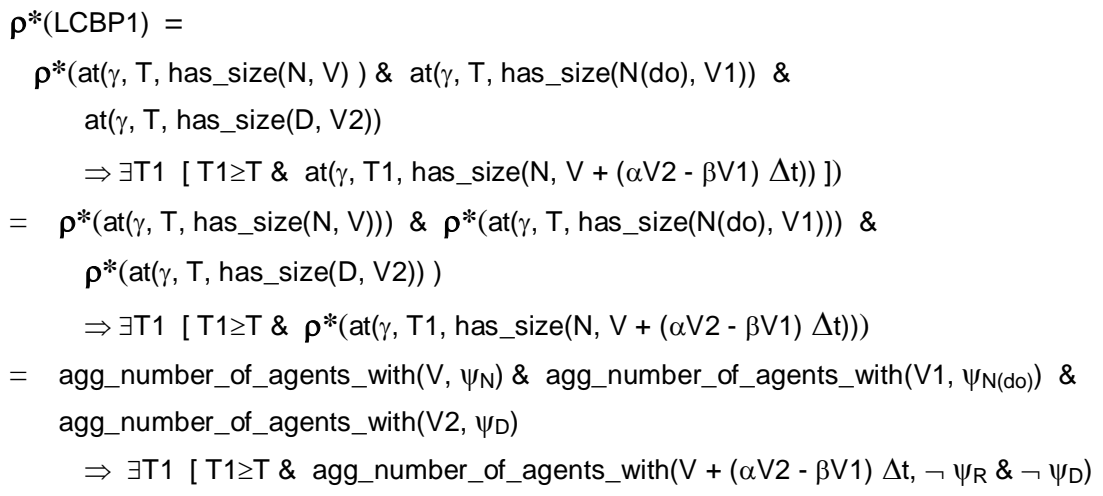

This property can be related by a (hybrid) logical entailment relation to the individual behavioural properties for the agents involved, for example, using an assumption on uniform distribution over time of the individual time points that dopamin lack becomes high. If this is a uniform fraction $\beta$ of $\mathrm{N}$ per time unit, then within $\Delta \mathrm{t}$ there are $\beta N \Delta t$ among them that start to have high dopamin lack. From the individual behavioural property BP1 for each of these agents it follows that within $\Delta \mathrm{t}$ they will generate a date request after $\Delta \mathrm{t}$; thus $\beta \mathrm{V} 1 \Delta \mathrm{t}$ date requests are generated, which makes these agents not part of $\mathrm{N}$ anymore, but of R. Similarly, assuming that dates have a fixed duration, it can be established that from group $\mathrm{D}$ a uniform fraction $\alpha$ will be make a transition to $\mathrm{N}$. Thus the third part of the interlevel relation for LCBP1 can be specified as

$$
\forall P[B P 1(P) \& B P 2(P) \& B P 3(P)] \Rightarrow \rho *(L C B P 1)
$$

This completes the interlevel relation specification for LCBP1. 


\section{Cognitive Cluster Interlevel Relations}

Also at the cognitive process abstraction level cluster interlevel relations can be established. As an example, the temporally local dynamic property LCCP1 connecting a collective desire to a collective intention.

\section{LCCP1 From collective desire to collective intention to request a date}

If the part $N($ desire(date)) of $N$ with desire to request a date has size $V$,

and the part $N($ intention(date_request $))$ of $N$ with intention to ask for a

date has size $V I$,

then the part $N($ intention(date_request $))$ of $N$ with intention to ask for

a date will have size $V+(V 1(V 1-V) / d) \Delta t$

at $(\gamma, T$, has_size $(\mathrm{N}($ desire(date $), \mathrm{V})) \&$ at $(\gamma, \mathrm{T}$, has_size $(\mathrm{N}($ intention(date_request $), \mathrm{V} 1))$

$\Rightarrow \exists T 1[T 1 \geq T \&$ at $(\gamma, T 1$, has_size $(\mathrm{N}($ intention(date_request), $\mathrm{V}+(\mathrm{V} 1(\mathrm{~V} 1-\mathrm{V}) / \mathrm{d}) \Delta \mathrm{t}))$

Roughly spoken this expresses that within cluster $\mathrm{N}$, a collective desire for a date within $\mathrm{N}$ affects the strength of a collective intention according to the formula $\mathrm{V}+(\mathrm{V} 1$ $(\mathrm{V} 1-\mathrm{V}) / \mathrm{d}) \Delta \mathrm{t}$. To map this higher level dynamic property onto an aggregation of lower level properties, first the ontology mapping $\rho$ for the collective temporal atoms at $(\gamma$, $\mathrm{T}$, has_size $(\mathrm{N}(\mathrm{C}), \mathrm{V})$ with $\mathrm{C}$ a cognitive concept is addressed (in this case $\mathrm{C}$ applies to a desire and an intention). In a manner similar to the approach above this mapping can be defined by

$$
\rho(\text { at }(\gamma, T \text {, has_size }(\mathrm{N}(\mathrm{C}), \mathrm{V})))=\text { agg_number_of_agents_with }(\mathrm{V}, \psi \mathrm{N}, \mathrm{C}(\mathrm{P}))
$$

with

$$
\psi_{\mathrm{N}, \mathrm{C}(\mathrm{P})}=\neg \psi_{\mathrm{R}} \& \neg \psi_{\mathrm{D}} \& \text { at }(\gamma, \mathrm{T}, \mathrm{C}(\mathrm{P})) .
$$

Here for $\mathrm{C}=$ intention $(X)$, the term $\mathrm{C}(\mathrm{P})$ denotes intention $(\mathrm{P}, \mathrm{X})$, and for $\mathrm{C}=\operatorname{desire}(\mathrm{X})$, the term $C(P)$ denotes desire $(P, X)$. Next the mapping is extended to dynamic property LCCP1:

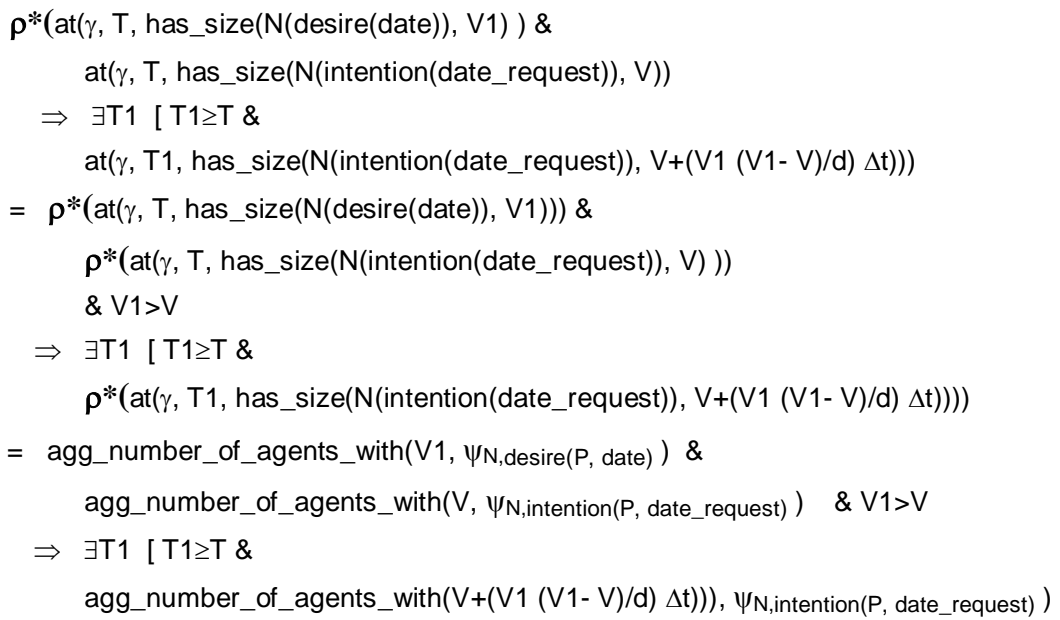

This aggregated lower level property, onto which the collective property was mapped, expresses how within cluster $\mathrm{N}$, the aggregated number of agents with a 
desire for a date affects the aggregated number of agents with the related intention. Indeed, in a manner similar to the behavioural case, such a property can be logically related to the temporally local dynamic property LCP2 at the cognitive process abstraction level that relates an individual desire to an individual intention, applied to the set of agents having this desire. Therefore the third part of the interlevel relation for LCCP1 can be specified as

$\forall P$ LCP2(P) $\Rightarrow \rho^{*}($ LCCP1).

This completes the interlevel relation specification for LCCP1.

\section{Neurological Cluster Interlevel Relations}

An interlevel relation at the neurological (or physiological) process abstraction level can be addressed according to an approach similar to the aggregation approach for the cognitive level. However, a difference is that here the individual states themselves have certain gradations or levels of activation. To take this into account in a mapping of collective activation states, two routes can be followed. The first route first makes the activation states per agent binary by considering those with activation level $\geq 0.5$, and aggregating (counting) only these agents. This route then resembles the approach for the cognitive process abstraction level. The second route takes the activation levels as a kind of weights in the aggregation process. This can be based on the following form of aggregation for the activation levels of $\mathrm{x}$ :

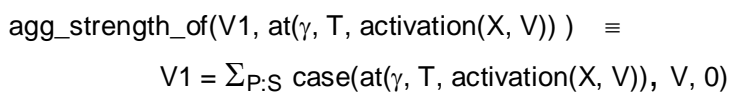

This takes as aggregated strength the sum of all activation values over all agents. The aggregated strength defined in this manner can be used in the mapping of the strength of the collective variant of the same neural state. Given this form of aggregation of activation levels, further the approach as for the cognitive level can be followed.

\section{Combining Dimensions and Commutation Principles}

Criterion (3) of an interpretation mapping

$$
T_{2} \vdash L \Rightarrow T_{1} \vdash \varphi^{*}(L)
$$

can be applied to a specification $L$ of a (e.g., emerging) pattern that is entailed by a model. Then it states that a pattern entailed by the higher level model is mapped onto a pattern entailed by the lower level model. In particular, it can be applied to valid logical implications that are part of an interlevel relation for a different dimension. For example, at a higher temporal level, consider a process abstraction interlevel relation from (multi-)agent model $\mathrm{AM}_{22}$ at the higher process abstraction level to 
(multi-)agent model $\mathrm{AM}_{21}$ at the lower process abstraction level specified by ontology mapping $\pi$ and a valid implication

$$
\mathrm{C} \Rightarrow \pi^{\star}(\mathrm{DP})
$$

with $\mathrm{C}$ a conjunction of dynamic properties from the model $\mathrm{AM}_{21}$, and DP a dynamic property of the model $\mathrm{AM}_{22}$. By applying a temporal interlevel relation from model $\mathrm{AM}_{21}$ to model $\mathrm{AM}_{11}$ based on $\theta$ this provides that the following is entailed by $\mathrm{AM}_{11}$ :

$$
\theta^{\star}\left(\mathrm{C} \Rightarrow \pi^{\star}(\mathrm{DP})\right)=\theta^{\star}(\mathrm{C}) \Rightarrow \theta^{*}\left(\pi^{\star}(\mathrm{DP})\right)=\mathrm{C}^{\prime} \Rightarrow \theta^{*}\left(\pi^{\star}(\mathrm{DP})\right)
$$

with $C^{\prime}$ a conjunction of properties entailed by the model $\mathrm{AM}_{11}$ at the lower temporal and lower process abstraction level. This can be reformulated into a specification of an entailment relation from properties of $\mathrm{AM}_{11}$ to $\theta^{*}\left(\pi^{\star}(\mathrm{DP})\right)$, as part of the process abstraction interlevel relation from model $\mathrm{AM}_{12}$ to $\mathrm{AM}_{11}$. These considerations indicate how interlevel relations for different dimensions may be combined and related; for a general picture, see Figure 8.

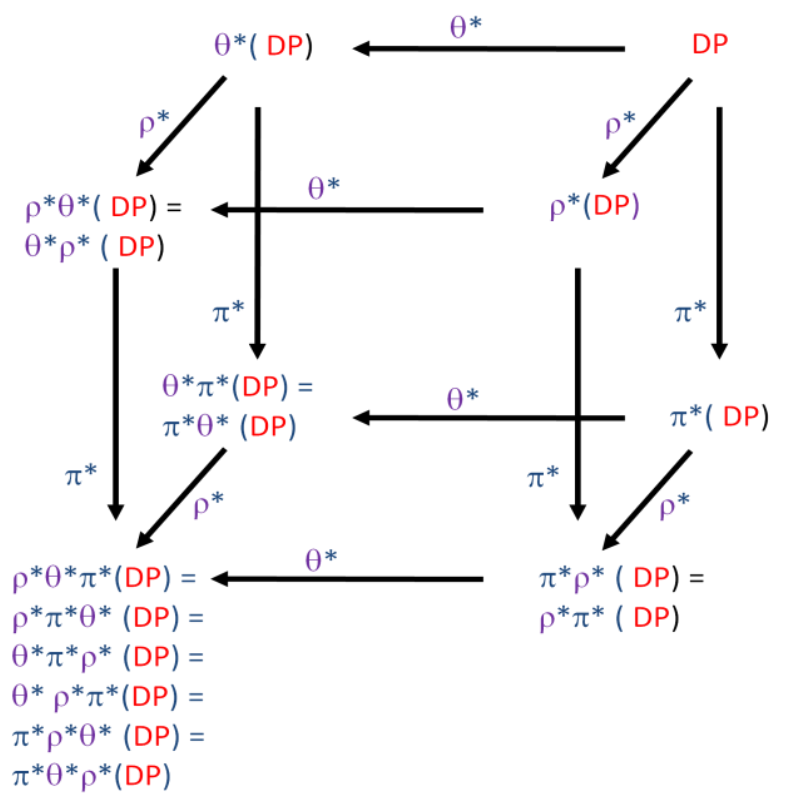

Figure 8. Combining interlevel relations and commutation principles

Note that here each of $\pi^{*}, \theta^{*}, \rho^{*}$ can be given indices $11,12,21,22$ to distinguish the four occurrences. This suggests heuristics to find interlevel relations from other interlevel relations, but one step further it suggests that interlevel relations may be specified in a coherent manner so that certain commutation rules hold, such as

$$
\pi_{12} *\left(\theta_{22} *(\mathrm{DP})\right)=\theta_{21} *\left(\pi_{22} *(\mathrm{DP})\right)
$$


or, if the tuples of four indexed mappings are indicated by one not indexed symbol, in the form of the following commutation rules (see also Section 4, Figure 6):

$\pi^{*} \theta^{*}=\theta^{*} \pi^{*}, \pi^{*} \rho^{*}=\rho^{*} \pi^{*}, \rho^{*} \theta^{*}=\theta^{*} \rho^{*}$.

Such commutation rules may not hold in any arbitrary setting, but given an assumption that the models describe the same reality (using different conceptualisations), they may be set up in a coherent manner so that these relationships do hold.

\section{Discussion}

The specification format for interlevel relations (e.g., [5]) introduced was inspired by the concept of interpretation mapping from logic to describe relations between logical theories (e.g., [28]), which has played a quite powerful role in mathematical logic and the logical foundations of mathematics. This concept has also been used to describe reduction relations between cognitive and neurological agent models (e.g., [20], [22], [29]). The work reported in the current paper generalises and applies this idea to a much wider spectrum of abstraction dimensions (taken from [8]). As a basis for the formalisation in a hybrid specification format reified sorted temporal predicate logic was used (e.g., [16]), but alternative choices may work equally well, for example, nonreified temporal predicate logic (using time arguments within each predicate), or any other temporal logic which is able to handle numbers. Moreover, the underlying conceptual and logical framework may well be related and combined with further work in ontology and database schema specification; e.g., [13].

Such interlevel relation specifications provide a useful conceptual and formal tool in analysis and design of multi-agent processes. For example, in the analysis of oneto-many negotiation processes (such as in [11]) it may be conceptually useful to be able to switch perspective between the 'many' role as many individual agents or as one super-agent (thus obtaining a conceptualisation as one-to-one negotiation), and to specify the relationship between the two conceptualisations. In the same analysis it may be useful to specify temporally global properties as a form of requirements (required emerging properties; for example, termination of the negotiation process) vs temporally local properties for mechanisms to realise these requirements (for example, the agents' decision rules). A temporal interlevel relation specification can be used to indicate which of the specified temporally local properties (are assumed to) realise which required temporally global properties. Many similar cases can be found in analysis and design of organisation models. In such cases, having models available at different abstraction levels, and knowing their relationships can provide a good basis to manage the complexity of the analysis and design process, both at an individual analyst/designer level, and in communication between different persons involved. At a more abstract level the main ideas can become and stay more transparent to everyone involved (including less technical stakeholders). One particular use is when a top down approach is followed in which first temporally 
global behavioural requirements are formulated for units consisting of larger groups of agents, and during the process these are (gradually) refined to temporally local properties for individual agents involving their internal cognitive and/or neurological dynamics (e.g., [12]). These show some possibilities for the perspective to use the presented hybrid (logical/numerical) formal specification framework to establish relationships between different multi-agent system models, for example, between behavioural, cognitive and neurological agent models (e.g., [4], [20], [22], [27], [29]), between emerging dynamic properties and mechanisms underlying them (e.g., [1], [2], [3], [6], [9], [10], [12], [15], [17], [19], [25], [26]), between population-based and individual agent-based models (e.g., [7], [21], [24], [30], [31]), and between organisation models and agent models (e.g., [14], [18], [23], [32]).

\section{References}

[1] Ashby, W.R.: Design for a Brain. Chapman \& Hall, ed. 1960.

[2] Baier, C., Katoen, J.-P.: Principles of Model Checking. MIT Press (2008)

[3] Barringer, H., Fisher, M., Gabbay, D., Owens, R., Reynolds, M.: The Imperative Future: Principles of Executable Temporal Logic, John Wiley \& Sons (1996)

[4] Baum, W. M. (1994). Understanding behaviorism: science, behavior, and culture. Harper Collins College Publishers.

[5] Boogerd, F., Bruggeman, F., Jonker, C.M., Looren de Jong, H., Tamminga, A., Treur, J., Westerhoff, H.V., and Wijngaards, W.C.A., Interlevel Relations in Computer Science, Biology, and Psychology. Philosophical Psychology 15, 2002, pp. 463-471.

[6] Bordini, R.H., Fisher, M., Visser, W., Wooldridge, M.: Verifying multi-agent programs by model checking. Auton Agent Multi-Agent Sys 12, 239--256 (2006)

[7] Bosse, T., Gerritsen, C., Hoogendoorn, M., Jaffry, S.W., and Treur, J., Agent-Based versus Population-Based Simulation of Displacement of Crime: A Comparative Study. Web Intelligence and Agent Systems Journal, vol. 9, 2011, pp. 147-160.

[8] Bosse, T., Hoogendoorn, M., Klein, M.C.A., and Treur, J., (2010). A Three-Dimensional Abstraction Framework to Compare Multi-Agent System Models. In: Pan, J.-S., Chen, S.M., and Nguyen, N.T. (eds.), Proc. of the Second Int. Conf. on Computational Collective Intelligence, ICCCI'10. Lecture Notes in Artificial Intelligence, vol. 6421, pp. 306-319, Springer Verlag, 2010.

[9] Bosse, T., Jonker, C.M., Meij, L. van der, Sharpanskykh, A., Treur, J.: Specification and Verification of Dynamics in Agent Models. Int. J. of Coop. Inf. Systems 18, 167-193 (2009)

[10] Bosse, T., Jonker, C.M., Meij, L. van der, Treur, J.: A Language and Environment for Analysis of Dynamics by Simulation. Intern. J. of AI Tools 16, 435-464 (2007)

[11] Brazier, F.M.T., Cornelissen, F., Gustavsson, R., Jonker, C.M., Lindeberg, O., Polak, B., and Treur, J., Compositional Verification of a Multi-Agent System for One-to-Many Negotiation. Journal of Applied Intelligence, vol. 20, 2004, pp. 95-117.

[12] Darimont, R., Lamsweerde, A. van: Formal Refinement Patterns for Goal-Driven Requirements Elaboration. Proc. of the 4th ACM Symposium FSE4, pp. 179-190 (1996)

[13] Euzenat, J., and Shvaiko, P., Ontology matching. Springer Verlag, 2007. 
[14] Ferber, J., Gutknecht, O., Jonker, C.M., Müller, J.P., Treur, J.: Organization Models and Behavioural Requirements Specification for Multi-Agent Systems In: Proc. of the 10th European Workshop MAAMAW'01 (2001).

[15] Forrester, J.W. (1961). Industrial Dynamics. Cambridge, MA, MIT Press, 1961.

[16] Galton, A., (2006). Operators vs Arguments: The Ins and Outs of Reification. Synthese, vol. 150 , pp. 415-441

[17] Jonker, C.M., Treur, J.: (2002). Compositional Verification of Multi-Agent Systems: a Formal Analysis of Pro-activeness and Reactiveness. Int J Coop Inf Sys 11, 51-92 (2002)

[18] Jonker, C.M., Treur, J., and Wijngaards, W.C.A., Specification, Analysis and Simulation of the Dynamics within an Organisation. Applied Intelligence, vol. 27, 2007, pp. 131152.

[19] Kelso, J.A.S.: Dynamic Patterns: The Self-Organization of Brain and Behavior. MIT Press (1995).

[20] Kim, J.: Philosophy of Mind. Westview Press (1996)

[21] Lovelock, J. (1979). Gaia: a new look at life on earth. Oxford University Press.

[22] Memon, Z.A., and Treur, J., Cognitive and Biological Agent Models for Emotion Reading. In: Proceedings of the 8th International Conference on Intelligent Agent Technology, IAT'08. IEEE Computer Society Press, 2008, pp. 308-313.

[23] Minsky, M., (1986). The Society of Mind. Simon and Schuster.

[24] Parunak, H.V.D., Savit, R., Riolo, R.L.: Agent-Based Modeling vs. Equation-Based Modeling: A Case Study and Users' Guide. In: J.S. Sichman et al. (eds.) MABS'98, LNAI, vol. 1534, pp. 10-25. Springer Verlag (1998)

[25] Port, R.F., Gelder, T. van (eds.): Mind as Motion: Explorations in the Dynamics of Cognition. MIT Press (1995)

[26] Roever, W.P. de, Langmaack, H., Pnueli A. (eds.), Compositionality - The Significant Difference, Proceedings of the International Workshop on Compositionality, COMPOS'97. LNCS, vol. 1536, Springer Verlag, 1998

[27] Sharpanskykh, A., and Treur, J., Relating Cognitive Process Models to Behavioural Models of Agents. In: Proceedings of the 8th International Conference on Intelligent Agent Technology, IAT'08. IEEE Computer Society Press, 2008, pp. 330-335.

[28] Tarski, A., Mostowski, A., and Robinson, R.M. (1953). Undecidable Theories. NorthHolland.

[29] Treur, J., On the Use of Reduction Relations to Relate Different Types of Agent Models. Web Intelligence and Agent Systems Journal, vol. 9, 2011, pp. 81-95.

[30] Volterra, V., Variations and fluctuations of the number of individuals in animal species living together. In: Animal Ecology. McGraw-Hill. Translated by R. N. Chapman. (1931)

[31] Wilson, W.G. (1998). Resolving Discrepancies between Deterministic Population Models and Individual-Based Simulations. American Naturalist, 151(2):116-134, 1998.

[32] Zambonelli, F., Jennings, N.R., Wooldridge, M.: Organisational Abstraction for the Analysis and Design of Multi-Agent Systems. In: Proc. AOSE 2000, LNCS vol. 1957, pp. 235-251. Springer (2001) 